\title{
A topological interpretation of the cyclotomic polynomial
}

\author{
Gregg Musiker $\llbracket$ and Victor Reiner 1 " \\ ${ }^{1}$ University of Minnesota, School of Mathematics, Minneapolis, MN, USA
}

\begin{abstract}
We interpret the coefficients of the cyclotomic polynomial in terms of simplicial homology.
Résumé. Nous donnons une interprétation des coefficients du polynôme cyclotomique en utilisant l'homologie simpliciale.
\end{abstract}

Keywords: Cyclotomic polynomial, higher-dimensional tree, matroid duality, oriented matroid, simplicial matroid, simplicial homology

\section{Introduction}

This paper studies the cyclotomic polynomial $\Phi_{n}(x)$, which is defined as the minimal polynomial over $\mathbb{Q}$ for any primitive $n^{\text {th }}$ root of unity $\zeta$ in $\mathbb{C}$. It is monic, irreducible, and has degree given by the Euler phi function $\phi(n)$, with formula

$$
\Phi_{n}(x)=\prod_{j \in(\mathbb{Z} / n \mathbb{Z})^{\times}}\left(x-\zeta^{j}\right) .
$$

The equation $x^{n}-1=\prod_{d \mid n} \Phi_{d}(x)$ gives a recurrence showing that all coefficients of $\Phi_{n}(x)$ lie in $\mathbb{Z}$.

Although well-studied, the coefficients of $\Phi_{n}(x)$ are mysterious [2, 10, 11, 14, 15, 17, 29]. We offer here two interpretations for their magnitudes, as orders of cyclic groups. In the first interpretation (Corollary 5 below) this group is a quotient of the free abelian group $\mathbb{Z}[\zeta]$ by a certain full rank sublattice.

The second interpretation is topological, given by Theorem 1 below, as the torsion in the homology of a certain simplicial complex associated with a squarefree integer $n=p_{1} \cdots p_{d}$. These simplicial complexes originally arose in the work of Bolker [6], reappeared in the work of Kalai [13] and Adin [1] on higherdimensional matrix-tree theorems, and were shown to be connected with cyclotomic extensions in work of J. Martin and the second author [18]. We review these simplicial complexes briefly here in order to state the result; see Section 4 for more details.

Given a positive integer $p$, let $K_{p}$ denote a 0 -dimensional abstract simplicial complex having $p$ vertices ${ }^{(i)}$, which we will label by the residues

$$
\{0 \bmod p, 1 \bmod p, \ldots,(p-1) \bmod p\}
$$

\footnotetext{
${ }^{\dagger}$ Supported by NSF Postdoctoral Fellowship and NSF grant DMS-1067183.

${ }^{\ddagger}$ Supported by NSF grant DMS-1001933.

(i) Note that here $K_{p}$ does not refer to a complete graph on $p$ vertices; we hope that this causes no confusion. 
for reasons that will become clear in a moment. Given primes $p_{1}, \ldots, p_{d}$, let

$$
K_{p_{1}, \ldots, p_{d}}:=K_{p_{1}} * \cdots * K_{p_{d}}
$$

be the simplicial join, [21, $\S 62]$, of $K_{p_{1}}, \ldots, K_{p_{d}}$. This is a pure $(d-1)$-dimensional abstract simplicial complex, that may be thought of as the complete $d$-partite complex on vertex sets $K_{p_{1}}$ through $K_{p_{d}}$ of sizes $p_{1}, \ldots, p_{d}$. The facets (maximal simplices) of $K_{p_{1}, \ldots, p_{d}}$ are labelled by sequences of residues $\left(j_{1} \bmod p_{1}, \ldots, j_{d} \bmod p_{d}\right)$. Denoting the squarefree product $p_{1} \cdots p_{d}$ by $n$, the Chinese Remainder Theorem isomorphism

$$
\mathbb{Z} / p_{1} \mathbb{Z} \times \cdots \times \mathbb{Z} / p_{d} \mathbb{Z} \stackrel{\Xi}{\longrightarrow} \mathbb{Z} / n \mathbb{Z}
$$

allows one to label such a facet by a residue $j \bmod n$; call this facet $F_{j \bmod n}$. Then for any subset $A \subseteq\{0,1, \ldots, \phi(n)\}$, let $K_{A}$ denote the subcomplex of $K_{p_{1}, \ldots, p_{d}}$ which is generated by the facets $\left\{F_{j \bmod n}\right\}$ as $j$ runs through the following set of residues:

$$
A \cup\{\phi(n)+1, \phi(n)+2, \ldots, n-1, n\} .
$$

Our first main result interprets the magnitudes of the coefficients of $\Phi_{n}(x)$. Let $\tilde{H}_{i}(-; \mathbb{Z})$ denote reduced simplicial homology with coefficients in $\mathbb{Z}$.

Theorem 1 For a squarefree positive integer $n=p_{1} \cdots p_{d}$, with cyclotomic polynomial $\Phi_{n}(x)=\sum_{j=0}^{\phi(n)} c_{j} x^{j}$, one has

$$
\tilde{H}_{i}\left(K_{\{j\}} ; \mathbb{Z}\right)= \begin{cases}\mathbb{Z} / c_{j} \mathbb{Z} & \text { if } i=d-2, \\ \mathbb{Z} & \text { if both } i=d-1 \text { and } c_{j}=0, \\ 0 & \text { otherwise. }\end{cases}
$$

We furthermore interpret topologically the signs of the coefficients in $\Phi_{n}(x)$. For this, we use oriented simplicial homology, and orient the facet $F_{j \bmod n}$ having $j \equiv j_{i} \bmod p_{i}$ for $i=1,2, \ldots, d$ as

$$
\left[F_{j}\right]=\left[F_{j \bmod n}\right]=\left[j_{1} \bmod p_{1}, \ldots j_{d} \bmod p_{d}\right] .
$$

Theorem 2 Fix a squarefree positive integer $n=p_{1} \cdots p_{d}$ with cyclotomic polynomial $\Phi_{n}(x)=\sum_{j=0}^{\phi(n)} c_{j} x^{j}$. Then for any $j \neq j^{\prime}$ such that $c_{j}, c_{j^{\prime}} \neq 0$, one has $\tilde{H}_{d-1}\left(K_{\left\{j, j^{\prime}\right\}} ; \mathbb{Z}\right) \cong \mathbb{Z}$, and any nonzero $(d-1)$-cycle $z=\sum_{\ell} b_{\ell}\left[F_{\ell}\right]$ in this homology group will have $b_{j}, b_{j^{\prime}} \neq 0$, with

$$
\frac{c_{j}}{c_{j^{\prime}}}=-\frac{b_{j^{\prime}}}{b_{j}}
$$

In particular, $c_{j}, c_{j^{\prime}}$ have the same sign if and only if $b_{j}, b_{j^{\prime}}$ have opposite signs.

Example 3 We illustrate these theorems for $n=15$. Here $d=2, p_{1}=3, p_{2}=5$, and $\phi(n)=2 \cdot 4=8$. The cyclotomic polynomial is

$$
\begin{aligned}
\Phi_{15}(x) & =1-x+x^{3}-x^{4}+x^{5}-x^{7}+x^{8} \\
& =(+1) \cdot\left(x^{0}+x^{3}+x^{5}+x^{8}\right)+(-1) \cdot\left(x^{1}+x^{4}+x^{7}\right)+0 \cdot\left(x^{2}+x^{6}\right) .
\end{aligned}
$$




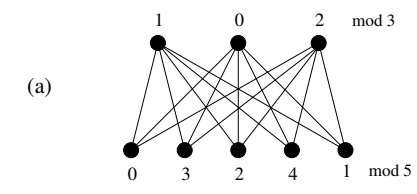

(b)

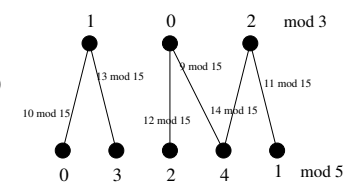

(c)

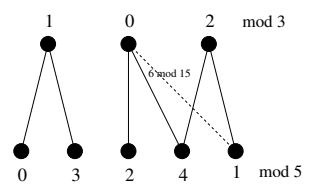

(d)

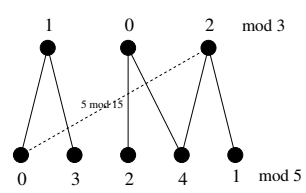

(e)

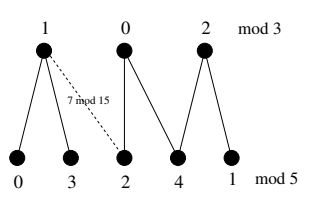

(f)

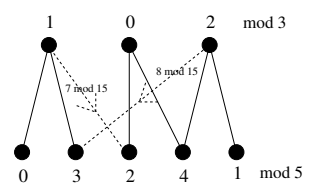

(g)

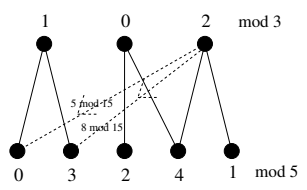

Fig. 1: The case of $\Phi_{15}(x)$

The complex $K_{p_{1}, p_{2}}=K_{3,5}$ is a complete bipartite graph with vertex sets labelled as in Figure 1(a). The subcomplex $K_{\varnothing}$ generated by the edges $F_{j \bmod 15}$ with $j \in\{\phi(n)+1, \phi(n)+2, \ldots, n-1\}=$ $\{9,10,11,12,13,14\}$ is the subgraph shown in Figure 1(b).

To see why the coefficient $c_{6}=0$ in $\Phi_{15}(x)$, one adds the edge $F_{6 \bmod 15}$ to the graph $K_{\varnothing}$, obtaining the graph $K_{\{6\}}$, shown in Figure 1(c), which has

$$
\begin{aligned}
& \tilde{H}_{0}\left(K_{\{6\}} ; \mathbb{Z}\right)=\mathbb{Z}=\mathbb{Z} / 0 \mathbb{Z} \\
& \tilde{H}_{1}\left(K_{\{6\}} ; \mathbb{Z}\right)=\mathbb{Z} .
\end{aligned}
$$

To see why the coefficients $c_{5}=+1$ or $c_{7}=-1$ have magnitude 1 , one adds the edge $F_{5}$ mod 15 or $F_{7 \text { mod } 15}$ to the graph $K_{\varnothing}$, obtaining the graphs $K_{\{5\}}$ or $K_{\{7\}}$ shown in Figures 1(d) and 1(e), which have

$$
\begin{aligned}
& \tilde{H}_{0}\left(K_{\{5\}} ; \mathbb{Z}\right)=0=\mathbb{Z} /(+1) \mathbb{Z} \\
& \tilde{H}_{0}\left(K_{\{7\}} ; \mathbb{Z}\right)=0=\mathbb{Z} /(-1) \mathbb{Z} .
\end{aligned}
$$

To understand the signs of the coefficients, note first that, by convention, $\Phi_{15}(x)$ is monic, so the coefficient $c_{8}=c_{\phi(n)}=+1$. Therefore any other coefficient $c_{j}$ should have sign

$$
\operatorname{sgn}\left(c_{j}\right)=\frac{\operatorname{sgn}\left(c_{j}\right)}{\operatorname{sgn}\left(c_{8}\right)}=-\frac{\operatorname{sgn}\left(b_{8}\right)}{\operatorname{sgn}\left(b_{j}\right)}
$$

where $z=\sum_{i} b_{i}\left[F_{i}\right]$ is a nontrivial cycle in $K_{\{j, 8\}}$, in which the edge $\left[F_{j}\right]$ is directed from the vertex $\left(j_{1} \bmod 3\right)$ toward the vertex $\left(j_{2} \bmod 5\right)$. As shown in Figures $1(\mathrm{f})$ and $1(\mathrm{~g})$, the nontrivial cycle in $K_{\{7,8\}}$ has $\left[F_{7}\right],\left[F_{8}\right]$ oriented in the same direction, explaining why $c_{7}=-1$, while the nontrivial cycle in $K_{\{5,8\}}$ has $\left[F_{5}\right],\left[F_{8}\right]$ oriented in the opposite direction, explaining why $c_{5}=+1$. 
The remainder of the paper is structured as follows. Section 2 describes our first interpretation for the cyclotomic polynomial, which applies much more generally to any monic polynomial in $\mathbb{Z}[x]$. Section 3 reviews some facts, underlying the main results, about duality of matroids, Plücker coordinates, and oriented matroids. Section 4 recalls results and establishes terminology on Kalai's higher dimensional spanning trees in a simplicial complex. Section 5 discusses further properties of the simplicial complex $K_{p_{1}, \ldots, p_{d}}$ whose subcomplexes appear in Theorem 1 and 2 Section 6 proves these theorems. We end with Section 7. where we discuss known properties of $\Phi_{n}(x)$ that manifest themselves topologically.

\section{Coefficients of monic polynomials in $\mathbb{Z}[x]$}

Our goal here is the first interpretation for the coefficients of $\Phi_{n}(x)$, which applies more generally to the coefficients of any monic polynomial $f(x)$ in $\mathbb{Z}[x]$. Recall that when $f(x)$ is of degree $r$, one has an isomorphism of $\mathbb{Z}$-modules

$$
\begin{aligned}
\mathbb{Z}^{r} & \longrightarrow \mathbb{Z}[x] /(f(x)) \\
\left(a_{0}, a_{1}, \ldots, a_{r-1}\right) & \longmapsto \sum_{j=0}^{r-1} a_{j} \bar{x}^{j} .
\end{aligned}
$$

As notation, given a subset $A$ of some abelian group, let $\mathbb{Z} A$ denote the collection of all $\mathbb{Z}$-linear combinations of elements of $A$.

Proposition 4 For a monic polynomial $f(x)=\sum_{j=0}^{r} c_{j} x^{j}$ of degree $r$ in $\mathbb{Z}[x]$, one has an isomorphism of abelian groups

$$
(\mathbb{Z}[x] /(f)) / \mathbb{Z} A \cong \mathbb{Z} / c_{j} \mathbb{Z}
$$

where $A$ is the subset of size $r$ given as $\left\{\overline{1}, \bar{x}, \overline{x^{2}}, \ldots, \overline{x^{r}}\right\} \backslash\left\{\overline{x^{j}}\right\}$.

Proof: Consider the matrix in $\mathbb{Z}^{r \times(r+1)}$

$$
\left[\begin{array}{cccccc}
1 & 0 & 0 & \cdots & 0 & -c_{0} \\
0 & 1 & 0 & \cdots & 0 & -c_{1} \\
0 & 0 & 1 & \cdots & 0 & -c_{2} \\
\vdots & \vdots & \vdots & & \vdots & \vdots \\
0 & 0 & 0 & \cdots & 1 & -c_{r-1}
\end{array}\right]
$$

whose columns express the elements of $\left\{\overline{1}, \bar{x}, \overline{x^{2}}, \ldots, \overline{x^{r}}\right\}$ uniquely in the $\mathbb{Z}$-basis $\left\{\overline{1}, \bar{x}, \overline{x^{2}}, \ldots, \overline{x^{r-1}}\right\}$ for $\mathbb{Z}[x] /(f)$. The $r \times r$ submatrix obtained by restricting this matrix to the columns indexed by $A$ is equivalent by row and column permutations to a upper triangular matrix with diagonal entries $\left(1,1, \ldots, 1,-c_{j}\right)$. Hence $(\mathbb{Z}[x] /(f)) / \mathbb{Z} A \cong \mathbb{Z} / c_{j} \mathbb{Z}$.

The special case where $f(x)$ is the cyclotomic polynomial $\Phi_{n}(x)$ leads to the following considerations. Fix once and for all a primitive $n^{\text {th }}$ root of unity $\zeta$.

Corollary 5 The cyclotomic polynomial $\Phi_{n}(x)=\sum_{j=0}^{\phi(n)} c_{j} x^{j}$ has

$$
\mathbb{Z}[\zeta] / \mathbb{Z} A \cong \mathbb{Z} / c_{j} \mathbb{Z}
$$

where $A=\left\{1, \zeta, \zeta^{2}, \ldots, \zeta^{\phi(n)}\right\} \backslash\left\{\zeta^{j}\right\}$. 
Proof: Apply the previous proposition with $f(x)=\Phi_{n}(x)$ and $r=\phi(n)$, noting that the ring map $\mathbb{Z}[x] \rightarrow \mathbb{Z}[\zeta]$ sending $x$ to $\zeta$ will also send $x^{j}$ to $\zeta^{j}$, and induce an isomorphism $\mathbb{Z}[x] /\left(\Phi_{n}(x)\right) \rightarrow \mathbb{Z}[\zeta]$.

For later use (see the proof of Theorem 20, we note here that the set

$$
P_{n}:=\left\{\zeta^{m}\right\}_{m \in(\mathbb{Z} / n \mathbb{Z})^{\times}}
$$

of all primitive $n^{\text {th }}$ roots of unity within $\mathbb{Z}[\zeta]$ forms a $\mathbb{Z}$-basis whenever $n$ is squarefree. This is a sharpening of an observation of Johnsen [12], who noted that $P_{n}$ forms a $\mathbb{Q}$-basis of $\mathbb{Q}[\zeta]$ in the same situation.

Proposition 6 When $n$ is squarefree, the collection $P_{n}$ of all primitive $n^{\text {th }}$ roots of unity forms a $\mathbb{Z}$-basis for $\mathbb{Z}[\zeta]$.

Proof: The result is easy when $n$ is prime and can be deduced from the Chinese Remainder Theorem in the general case. See [22] for details.

\section{Duality of matroids or Plücker coordinates}

We will need a version of the linear algebraic duality between Plücker coordinates for complementary Grassmannians $G\left(r, \mathbb{F}^{n}\right), G\left(n-r, \mathbb{F}^{n}\right)$, or equivalently, the duality between bases and cobases in coordinatized matroids.

Proposition 7 Let $0 \leq r \leq n$. Let $M$ and $M^{\perp}$ be matrices in $\mathbb{F}^{r \times n}$ and $\mathbb{F}^{(n-r) \times n}$, respectively, both of maximal rank, with the following property: $\operatorname{ker} M$ is equal to the row space of $M^{\perp}$, or equivalently, ker $M^{\perp}$ is the row space of $M$. Then

(i) there exists a scalar $\alpha$ in $\mathbb{F}^{\times}$having the following property: for every $(n-r)$-subset $T$ of $[n]$, with complementary set $T^{c}$,

$$
\operatorname{det}\left(\left.M\right|_{T^{c}}\right)= \pm \alpha \cdot \operatorname{det}\left(\left.M^{\perp}\right|_{T}\right)
$$

where $\left.A\right|_{J}$ denotes the restriction of a matrix $A$ to the subset of columns indexed by $J$, and the \pm sign depends upon the set $T$.

(ii) if one furthermore assumes that $\mathbb{F}=\mathbb{Q}$, that $M$ and $M^{\perp}$ have entries in $\mathbb{Z}$, and that there exists at least one $(n-r)$-subset $T_{0}$ for which $\left.M\right|_{T_{0}^{c}},\left.M^{\perp}\right|_{T_{0}}$ are both invertible over $\mathbb{Z}$, then the scalar $\alpha$ above equals \pm 1 , and one has for every $(n-r)$-subset $T$,

$$
\operatorname{coker}\left(\left.M\right|_{T^{c}}\right) \cong \operatorname{coker}\left(\left.M^{\perp}\right|_{T}\right) \text {. }
$$

Here, we are thinking of coker $M$ as signifying a map between powers of $\mathbb{Z}$.

Proof: Both assertions can be reduced via row and column operations to the case where $M$ takes the form $\left[I_{r} \mid A\right]$ for some $r$-by- $(n-r)$ matrix $A$, where they are easier to verify. See [22] for details.

The proof of Theorem 2 will ultimately rely on the following statement about duality of oriented matroids for vectors in a vector space over an ordered field $\mathbb{F}$, such as $\mathbb{F}=\mathbb{Q}$. 
Proposition 8 Let $\mathbb{F}$ be an ordered field, $M$ and let $M^{\perp}$ be matrices in $\mathbb{F}^{r \times n}$ and $\mathbb{F}^{(n-r) \times n}$ as in Proposition 7 that is, both of maximal rank, with $\operatorname{ker} M$ perpendicular to the row space of $M^{\perp}$. Let the vectors $v_{\ell}$ in $\mathbb{F}^{r}$ and $v_{\ell}^{\perp}$ in $\mathbb{F}^{n-r}$ be the $\ell^{\text {th }}$ columns of $M$ and $M^{\perp}$. Let $A$ be an $(r+1)$-subset of $\{1,2, \ldots, n\}$ such that the matrix $\left.M\right|_{A}$ in $\mathbb{F}^{r \times(r+1)}$ has full rank $r$, with

$$
\sum_{\ell \in A} c_{\ell} v_{\ell}=0
$$

the unique dependence among its columns, up to scaling. Then for any pair of nonzero coefficients $c_{j}, c_{j^{\prime}} \neq 0$, the matrix $\left.M^{\perp}\right|_{A^{c} \cup\left\{j, j^{\prime}\right\}}$ in $\mathbb{F}^{(n-r) \times(n-r+1)}$ has full rank $n-r$, and the unique dependence among its columns, up to scaling,

$$
\sum_{\ell \in A^{c} \cup\left\{j, j^{\prime}\right\}} b_{\ell} v_{\ell}^{\perp}=0
$$

will have both $b_{j}, b_{j^{\prime}} \neq 0$, with

$$
\frac{c_{j}}{c_{j^{\prime}}}=-\frac{b_{j^{\prime}}}{b_{j}}
$$

In particular, $c_{j}, c_{j^{\prime}}$ have the same sign if and only if $b_{j}, b_{j^{\prime}}$ have opposite signs.

Proof: The main observation here is that vectors in the row space of $M^{\perp}$ are covectors for $\left\{v_{\ell}^{\perp}\right\}$. See [22] for details.

\section{Simplicial spanning trees}

For a collection of subsets $S$ of some vertex set $V$, let $\langle S\rangle$ denote the (abstract) simplicial complex $S$ on $V$ generated by $S$, that is, $\langle S\rangle \subset 2^{V}$ consists of all subsets of $V$ contained in at least one subset from $S$. We recall the notion of a simplicial spanning tree in $S$, following Adin [1], Duval, Klivans and Martin [8], Kalai [13], and Maxwell [19].

Definition 9 Let $S$ be the collection of facets of a pure $k$-dimensional (abstract) simplicial complex. Say that $R \subset S$ is an $S$-spanning tree if

(i) $\langle R\rangle$ contains the entire $(k-1)$-skeleton of $\langle S\rangle$,

(ii) $\tilde{H}_{k}(\langle R\rangle ; \mathbb{Z})=0$, and

(iii) $\tilde{H}_{k-1}(\langle R\rangle ; \mathbb{Z})$ is finite.

We point out here three well-known features of this definition.

Proposition 10 Fix the collection of facets $S$ of a pure k-dimensional simplicial complex.

(i) Condition (i) in Definition 9 is equivalent to $\tilde{H}_{k}(\langle S\rangle,\langle R\rangle ; \mathbb{Z})=\mathbb{Z}^{|S \backslash R|}$.

(ii) Condition (ii) in Definition 9 is equivalent to $\tilde{H}_{k}(\langle R\rangle ; \mathbb{Q})=0$. 
(iii) All S-spanning trees $R$ have the same cardinality, namely

$$
|R|=|S|-\operatorname{rank}_{\mathbb{Z}} \tilde{H}_{k}(\langle S\rangle ; \mathbb{Z})
$$

Proof: See [22] for details.

The following key observation essentially goes back to work of Kalai [13, Lemma 2].

Proposition 11 Fix a vertex set $V$ and a collection of $k$-dimensional simplices $S$. Consider a collection of $(k+1)$-dimensional faces $T$ of cardinality

$$
|T|:=\operatorname{rank}_{\mathbb{Z}} \tilde{H}_{k}(\langle S\rangle ; \mathbb{Z})
$$

for which $T \cup\langle S\rangle$ forms a simplicial complex $K$, that is, all boundaries of faces in $T$ lie in $\langle S\rangle$.

Then the following two assertions hold for any choice of an $S$-spanning tree $R$.

(i) The $|T| \times|T|$ matrix $\partial$ that represents the relative simplicial boundary map

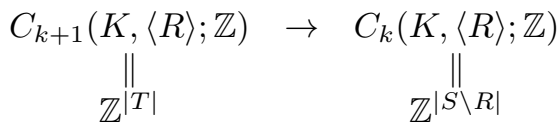

is nonsingular if and only if $\tilde{H}_{k+1}(K ; \mathbb{Q})=0$.

(ii) When the matrix $\partial$ is nonsingular, then $\operatorname{coker}(\partial)=\tilde{H}_{k}(K,\langle R\rangle ; \mathbb{Z})$.

Proof: See [22] for details.

Definition 12 Given a collection of $k$-simplices $S$, and an $S$-spanning tree $R$, say ${ }^{\text {(ii) }}$ that $R$ is torsion-free if Condition (iii) in Definition 9 is strengthened to the vanishing condition

(iv) $\tilde{H}_{k-1}(\langle R\rangle ; \mathbb{Z})=0$.

Example 13 For example, when $\langle R\rangle$ is a contractible subcomplex of $\langle S\rangle$ then it satisfies Condition (ii) of Definition 9 as well as the vanishing condition (iv). If it furthermore satisfies Condition (i) of Definition 9 . then $R$ becomes a torsion-free $S$-spanning tree.

A frequent combinatorial setting where this occurs (such as in Proposition 15 below) is when $S$ is the set of facets of a (pure) shellable [3] simplicial complex, and $R$ is the subset of facets which are not fully attached along their entire boundaries during the shelling process.

Proposition 14 Using the hypotheses and notation of Proposition 11, if one assumes in addition that $R$ is torsion-free, assertion (ii) of Proposition 11 becomes the following assertion about (non-relative) homology:

(ii) When the matrix $\partial$ is nonsingular, then $\operatorname{coker}(\partial)=\tilde{H}_{k}(K ; \mathbb{Z})$

Proof: When $R$ is torsion-free, the long exact sequence for the pair $(K,\langle R\rangle)$ shows that $\tilde{H}_{k}(K ; \mathbb{Z}) \cong$ $\tilde{H}_{k}(K,\langle R\rangle ; \mathbb{Z})$.

(ii) This condition on an $S$-spanning tree also plays an important role in [9] by Duval, Klivans and Martin. 


\section{More on the complete $d$-partite complex}

It is well-known and easy to see that for a positive integer $n$ having prime factorization $n=p_{1}^{e_{1}} \cdots p_{d}^{e_{d}}$ with $e_{i} \geq 1$, one always has $\Phi_{n}(x)=\Phi_{p_{1} \cdots p_{d}}\left(x^{n / p_{1} \cdots p_{d}}\right)$. Thus it suffices to interpret the coefficients of cyclotomic polynomials for squarefree $n$.

In this section, we fix such a squarefree $n=p_{1} \cdots p_{d}$, and discuss further properties of the simplicial complexes $K_{p_{1}, \ldots, p_{d}}$, defined in Section 1 , appearing in Theorems 1 and 2

Proposition 15 The $(d-2)$-dimensional skeleton of $K_{p_{1}, \ldots, p_{d}}$ is shellable, with

$$
\tilde{H}_{d-2}\left(K_{p_{1}, \ldots, p_{d}} ; \mathbb{Z}\right)=\mathbb{Z}^{n-\phi(n)} .
$$

Proof: To show that the $(d-2)$-skeleton is shellable, we note the following three facts: (i) zerodimensional complexes are all trivially shellable, (ii) joins of shellable complexes are shellable [24, Sec. 2], and (iii) skeleta of (pure) shellable simplicial complexes are shellable [5, Corollary 10.12]. Having shown that this skeleton is shellable, it therefore has only top homology; see, for example [3, Appendix]. This homology is free abelian, with rank the absolute value of its reduced Euler characteristic, namely

$$
\begin{aligned}
\left|\sum_{i \geq-1}(-1)^{i} \operatorname{rank}_{\mathbb{Z}}\left(C_{i}\right)\right| & =\left|\sum_{i \geq-1}(-1)^{i} \sum_{\substack{I \subsetneq\{1,2, \ldots, d\} \\
|I|=i+1}} \prod_{i \in I} p_{i}\right|=\left|\sum_{I \subsetneq\{1,2, \ldots, d\}}(-1)^{|I|-1} \prod_{i \in I} p_{i}\right| \\
& =\left|\left(p_{1}-1\right) \cdots\left(p_{d}-1\right)-p_{1} \cdots p_{d}\right|=|\phi(n)-n| .
\end{aligned}
$$

As noted in the introduction, the Chinese Remainder Theorem isomorphism (11) identifies elements of $\mathbb{Z} / n \mathbb{Z}$ with the $(d-1)$-dimensional simplices of $K_{p_{1}, \ldots, p_{d}}$. Lower dimensional faces of $K_{p_{1}, \ldots, p_{d}}$ can also be identified as cosets of subgroups within $\mathbb{Z} / n \mathbb{Z}$, but we will use this identification sparingly in this paper. For the sake of writing down oriented simplicial boundary maps, choose the following orientation on the simplices of $K_{p_{1}, \ldots, p_{d}}$, consistent with the orientation of facets preceding Theorem 2 . choose the oriented $(\ell-1)$-simplex $\left[j_{i_{1}} \bmod p_{i_{1}}, \ldots, j_{i_{\ell}} \bmod p_{i_{\ell}}\right]$ with $i_{1}<\ldots<i_{\ell}$ as a basis element of $C_{\ell-1}\left(K_{p_{1}, \ldots, p_{d}} ; \mathbb{Z}\right)$. The following simple observation was the crux of the results in [18].

Proposition 16 If one identifies the indexing set $\mathbb{Z} / n \mathbb{Z}$ for the columns of the boundary map

$$
C_{d-1}\left(K_{p_{1}, \ldots, p_{d}} ; \mathbb{Z}\right) \rightarrow C_{d-2}\left(K_{p_{1}, \ldots, p_{d}} ; \mathbb{Z}\right)
$$

with the set $\mu_{n}:=\left\{\zeta^{j}\right\}_{j \in \mathbb{Z} / n \mathbb{Z}}$ of all $n^{\text {th }}$ roots of unity, then every row of this boundary map represents a $\mathbb{Q}$-linear dependence on $\mu_{n}$.

Proof: A row in this boundary map is indexed by an oriented $(d-2)$-face, which has the form

$$
\left[j_{1} \bmod p_{1}, \ldots, j_{k} \widehat{\bmod } p_{k}, \ldots, j_{d} \bmod p_{d}\right]
$$

for some $j_{k} \in\left\{0,1, \ldots, p_{k}-1\right\}$ and $1 \leq k \leq d$. This row will contain mostly zeroes. Its non-zero entries are all $(-1)^{k-1}$, and lie in the columns indexed by those $\zeta^{j}$ having $j \equiv j_{i} \bmod p_{i}$ for $i \neq k$, and $j \bmod p_{k}$ arbitrary. These exponents $j$ are exactly those lying in one coset of the subgroup $p_{1} \cdots \hat{p_{k}} \cdots p_{d} \mathbb{Z} / n \mathbb{Z}$ within $\mathbb{Z} / n \mathbb{Z}$. Summing $\zeta^{j}$ over $j$ in such a coset gives zero. 
Example 17 Let $n=15$ as in Example 3, and consider the matrix for the simplicial boundary map $C_{1}\left(K_{3,5} ; \mathbb{Z}\right) \rightarrow C_{0}\left(K_{3,5} ; \mathbb{Z}\right)$. One of its rows is indexed by the 0 -face $[2 \bmod 5]$ and this row has exactly three nonzero entries, all equal to $(-1)^{0}=+1$. To see these signs, we rewrite [2 $\left.\bmod 5\right]$ in three ways, all of which involve deleting the first entry out of two in an oriented 1-face:

$$
[2 \bmod 5]=[0 \widehat{\bmod } 3,2 \bmod 5]=[1 \widehat{\bmod } 3,2 \bmod 5]=[2 \widehat{\bmod } 3,2 \bmod 5] .
$$

The columns corresponding to these three 1 -faces are indexed by the roots of unity $\zeta^{12}, \zeta^{7}$, and $\zeta^{2}$, respectively. Summing these up with coefficients of positive one, we get

$$
1 \cdot \zeta^{12}+1 \cdot \zeta^{7}+1 \cdot \zeta^{2}=\zeta^{2}\left(\zeta^{10}+\zeta^{5}+1\right)
$$

which is the sum of $\zeta^{j}$ over $j$ lying in a coset of $5 \mathbb{Z} / 15 \mathbb{Z}$, and hence is zero.

Definition 18 Assume that $n$ is squarefree and let $T$ denote any set of $n-\phi(n)$ columns of the boundary map (5). Identify the complementary set $T^{c}$ of $\phi(n)$ columns with a subset of the $n^{t h}$ roots-of-unity $\mu_{n}$. Create a subcomplex of $K_{p_{1}, \ldots, p_{d}}$ by including its entire $(d-2)$-skeleton and attaching the subset of $(d-1)$-faces indexed by $T$. We denote this subcomplex as $K[T]$.

With this definition in mind, we will make use of an interesting feature of this labelling of the boundary map and the set $P_{n}$ of primitive $n^{\text {th }}$ roots of unity, noted already in [18, Remark 5]. For this next result, we let $P_{n}^{c}$ denote the $(n-\phi(n))$-element subset of $\mu_{n}$ indexed by the $n^{\text {th }}$ roots of unity which are not primitive.

Proposition 19 Let $n$ be a squarefree integer and $P_{n}^{c}$ be as above. Then the subcomplex $K\left[P_{n}{ }^{c}\right]$ of $K_{p_{1}, \ldots, p_{d}}$ is contractible.

Proof: Observe that the primitive roots in $\mathbb{Z} / n \mathbb{Z}$ are exactly those elements which do not vanish modulo $p_{i}$ for $i=1, \ldots, d$. Tracing through the labelling of the $(d-1)$-faces via $\Xi$, we obtain the description

$$
K\left[P_{n}{ }^{c}\right]=\bigcup_{i=0}^{d} \operatorname{star}_{K_{p_{1}, \ldots, p_{d}}}\left(0 \bmod p_{i}\right),
$$

where $\operatorname{star}_{\Delta}(v)$ denotes the simplicial star of the vertex $v$ inside a simplicial complex $\Delta$. Furthermore, each intersection of these stars is nonempty and contractible, because it is the star of another face: for $I \subset[d]$,

$$
\bigcap_{i \in I} \operatorname{star}_{K_{p_{1}, \ldots, p_{d}}}\left(0 \bmod p_{i}\right)=\operatorname{star}_{K_{p_{1}, \ldots, p_{d}}}\left(\left\{0 \bmod p_{i}\right\}_{i \in I}\right) .
$$

A standard nerve lemma [4, Theorem 10.6] then shows that $K\left[P_{n}{ }^{c}\right]$ itself is contractible.

Theorem 20 Let $n$ be a squarefree integer and $T$ be a subset of $\mu_{n}$ of size $n-\phi(n)$. Let $K[T]$ be the subcomplex of $K_{p_{1}, \ldots, p_{d}}$ of Definition 18. Then

$$
\tilde{H}_{i}(K[T] ; \mathbb{Z}) \cong \begin{cases}\mathbb{Z}[\zeta] / \mathbb{Z} T^{c} & \text { if } i=d-2, \\ \mathbb{Z} & \text { if both } i=d-1 \text { and } \operatorname{rank}_{\mathbb{Z}}\left(\mathbb{Z} T^{c}\right)<\phi(n), \\ 0 & \text { otherwise. }\end{cases}
$$

where $\mathbb{Z} T^{c}$ is the sublattice $\mathbb{Z}$-spanned by the roots-of-unity $T^{c} \subset \mu_{n}$. 
Proof: Choose any $\mathbb{Z}$-basis for $\mathbb{Z}[\zeta]$. Let $M$ in $\mathbb{Z}^{\phi(n) \times n}$ be the matrix that expresses the $n^{\text {th }}$ roots of unity $\mu_{n}$ in this basis. We construct a particular matrix $M^{\perp}$ to accompany $M$ as in Proposition 7 part (ii). Consider the collection $S$ of all $(d-2)$-faces in the complete $d$-partite complex $K_{p_{1}, \ldots, p_{d}}$. The complex $\langle S\rangle$ generated by $S$ is therefore the $(d-2)$-skeleton of $K_{p_{1}, \ldots, p_{d}}$. Proposition 15 implies that $\langle S\rangle$ is shellable, and that it has $\operatorname{rank}_{\mathbb{Z}} \tilde{H}_{d-2}(\langle S\rangle ; \mathbb{Z})=n-\phi(n)$. Therefore, we are in the situation of Example 13 implying that there exists a torsion-free $S$-spanning tree $R$, and any such $R$ will have $|S \backslash R|=n-\phi(n)$.

Our candidate for the matrix $M^{\perp}$ in $\mathbb{Z}^{(n-\phi(n)) \times n}$ is the restriction of the boundary map from (5) to its rows indexed by $S \backslash R$. Proposition 16 shows that the rows of $M^{\perp}$ are all perpendicular to the rows of $M$. Now choose $T, T^{c}$ so that $T^{c}$ indexes the set $P_{n}$ of primitive $n^{\text {th }}$ roots of unity. Proposition 6 implies that the maximal minor $\left.M\right|_{T^{c}}$ of $M$ is invertible over $\mathbb{Z}$, while Proposition 19 implies that the maximal minor $\left.M^{\perp}\right|_{T}$ of $M^{\perp}$ is invertible over $\mathbb{Z}$. Thus $M, M^{\perp}$ satisfy the hypotheses of Proposition 7 part (ii), and combining this with Proposition 14 gives the assertion of the theorem.

\section{Proof of Theorems 1 and 2}

We are now in a position to prove Theorems 1 and 2

Proof of Theorem 1: Let $T^{c}=\left\{1, \zeta, \zeta^{2}, \ldots, \zeta^{\phi(n)}\right\} \backslash\left\{\zeta^{j}\right\}$ so that we have the equality of complexes $K[T]=K\left[\left\{\zeta^{\phi(n)+1}, \zeta^{\phi(n)+2}, \ldots, \zeta^{n-1}\right\} \cup\{j\}\right]=K_{j}$. The theorem then follows from Theorem 20 and Corollary 5 .

Proof of Theorem 2: We prove Theorem 2 by applying Proposition 8 to the matrices $M, M^{\perp}$ in the proof of Theorem 20. with $A=\left\{1, \zeta, \zeta^{2}, \ldots, \zeta^{\phi(n)}\right\}$. The dependence (2) among the columns of $\left.M\right|_{A}$ has the same coefficients (up to scaling) as the cyclotomic polynomial, and the dependence (3) among the columns of $\left.M^{\perp}\right|_{A^{c} \cup\left\{j, j^{\prime}\right\}}$ has the same coefficients (up to scaling) as a nonzero cycle $z=\sum_{\ell} b_{\ell}\left[F_{\ell}\right]$ in $\tilde{H}_{d-1}\left(K_{\left\{j, j^{\prime}\right\}} ; \mathbb{Z}\right)$.

\section{Concordance with known properties of $\Phi_{n}(x)$}

Here are some results about $\Phi_{n}(x)$ that manifest themselves topologically. See [22] for details.

1. The two maps $\mathbb{Z} / n \mathbb{Z} \rightarrow \mathbb{Z} / n \mathbb{Z}$ that send $\bar{j}$ to $\overline{-j}$ and send $\bar{j}$ to $\overline{j+1}$ generate a dihedral group of simplicial automorphisms of $K_{p_{1}, p_{2}, \ldots, p_{d}}$. One such automorphism sends the subcomplex $K_{\{j\}}$ to $K_{\{\phi(n)-j\}}$, and the subcomplex $K_{\{j, \phi(n)\}}$ to $K_{\{0, \phi(n)-j\}}$, explaining the symmetry $c_{j}=c_{\phi(n)-j}$ in $\Phi_{n}(x)$.

2. The fact that $\Phi_{2 n}(x)=\Phi_{n}(-x)$ when $n$ is odd manifests itself topologically as follows: the subcomplex $K_{\{j\}}$ whose homology interprets the coefficient of $x^{j}$ for $\Phi_{2 n}(x)$ is homotopy-equivalent to the suspension of the corresponding complex for $\Phi_{n}(x)$. Furthermore, there is a similar suspension relation between the complexes that predict the coefficients' signs. 
3. When $d=2$ so $n=p_{1} p_{2}$ is the product of only two primes, all the subcomplexes $K_{\{j\}}$ of $K_{p_{1}, p_{2}}$ are graphs. Hence their $(d-2)$-dimensional homology is torsion-free. It follows that the only nonzero coefficients of $\Phi_{n}(x)$ are \pm 1 , agreeing with a well-known old observation of Migotti [20]. The explicit expansion of $\Phi_{p_{1} p_{2}}(x)$ is given in Elder [10], Lam and Leung [16], and Lenstra [17].

4. In contrast to above, when $d \geq 3$ and the $p_{i}$ 's are odd primes, $\Phi_{n}(x)$ often has coefficients with absolute value $\geq 2$. For example, $\Phi_{105}(x)$ has coefficient -2 on $x^{7}$ and $x^{41}$. The 2 -dimensional subcomplexes $K_{\{7\}}$ and $K_{\{41\}}$, whose 1-homology equals $\mathbb{Z} / 2 \mathbb{Z}$, turn out to be surprisingly nontrivial. For example, neither one can be collapsed down to a real projective plane.

\section{Acknowledgements}

The authors wish to credit Jeremy Martin for the original observation that the cyclotomic matroid $\mu_{n}$ with $n=p_{1} p_{2}$ is cographic. They also thank Sam Elder, Nathan Kaplan, and Pieter Moree for helpful references on cyclotomic polynomials. This submission is an abridged version of [22]. We thank the referees for helpful editorial suggestions.

\section{References}

[1] R.M. Adin, Counting colorful multi-dimensional trees. Combinatorica 12 (1992), 247-260.

[2] G. Bachman, On the Coefficients of Cyclotomic Polynomials Memoirs of the American Mathematical Society 106, 1993.

[3] A. Björner, Shellable and Cohen-Macaulay partially ordered sets. Trans. Amer. Math. Soc. 260 (1980), no. 1, 159-183.

[4] A. Björner, Topological methods. Handbook of combinatorics, Vol. II, 1819-1872. Elsevier, Amsterdam, 1995

[5] A. Björner and M. Wachs, Shellable nonpure complexes and posets. II. Trans. Amer. Math. Soc. 349 (1997), no. 10, 3945-3975.

[6] E.D. Bolker, Simplicial geometry and transportation polytopes. Trans. Amer. Math. Soc. 217 (1976), $121-142$.

[7] H.H. Crapo and G.-C. Rota, On the foundations of combinatorial theory: Combinatorial geometries. Preliminary edition. The M.I.T. Press, Cambridge, Mass.-London, 1970.

[8] A.M. Duval, C.J. Klivans, and J.L Martin, Simplicial matrix-tree theorems. Trans. Amer. Math. Soc. 361 (2009), no. 11, 6073-6114.

[9] A.M. Duval, C.J. Klivans, and J.L Martin, Critical groups of simplicial complexes. preprint, arXiv:1101.3981

[10] S. Elder, Flat Cyclotomic Polynomials, Colorado Math Circle Talk, January 2, 2010, http://www.coloradomath.org/handouts/flat_cyclotomic.pdf 
[11] Y. Gallot and P. Moree, Neighboring ternary cyclotomic coefficients differ by at most one. $J . R a-$ manujan Math. Soc. 24 (2009), no. 3, 235248.

[12] K. Johnsen, Lineare Abhängigkeiten von Einheitswurzeln. Elem. Math. 40 (1985), 57-59.

[13] G. Kalai, Enumeration of $\mathbb{Q}$-acyclic simplicial complexes. Israel J. Math. 45 (1983), 337-351.

[14] N. Kaplan, Flat cyclotomic polynomials of order three. J. Number Theory 127 (2007), no. 1, 118 126.

[15] G. S. Kazandzidis, On the cyclotomic polynomial: Coefficients. Bull. Soc. Math. Gréce (N.S.) 4 (1963), no. 1, 1-11.

[16] T.Y. Lam and K.H. Leung, On the Cyclotomic Polynomial $\Phi_{p q}(X)$. Amer. Math. Monthly 103 (1996), 562-564.

[17] H.W. Lenstra, Vanishing sums of roots of unity, in: Proceedings, Bicentennial Congress Wiskundig Genootschap, Vrije Univ., Amsterdam, 1978, Part II, 1979, pp. 249-268.

[18] J. Martin and V. Reiner, Cyclotomic and simplicial matroids. Israel J. Math. 150 (2005), 229-240.

[19] M. Maxwell, Enumerating bases of self-dual matroids. J. Combin. Theory Ser. A 116 (2009), no. 2, 351-378.

[20] A. Migotti, Zur Theorie der Kreisteilungsgleichung. Sitzber. Math.-Naturwiss. Classe der Kaiser. Akad. der Wiss., Wien 87 (1883), 7-14.

[21] J.R. Munkres, Elements of algebraic topology. Addison-Wesley Publishing Company, Menlo Park, CA, 1984.

[22] G. Musiker and V. Reiner, The cyclotomic polynomial topologically. preprint, arXiv: 1012.1844

[23] J.G. Oxley, Matroid theory. Oxford Science Publications. The Clarendon Press, Oxford University Press, New York, 1992.

[24] J.S. Provan and L.J. Billera, Decompositions of simplicial complexes related to diameters of convex polyhedra. Math. Oper. Res. 5 (1980), no. 4, 576-594.

[25] D.J.A. Welsh, Matroid theory. London Math. Soc. Monographs 8. Academic Press, London-New York, 1976.

[26] N. White, Theory of matroids. Encyclopedia of Mathematics and its Applications 26. Cambridge University Press, Cambridge, 1986.

[27] N. White, Combinatorial geometries. Encyclopedia of Mathematics and its Applications 29. Cambridge University Press, Cambridge, 1987.

[28] N. White, Matroid applications. Encyclopedia of Mathematics and its Applications 40. Cambridge University Press, Cambridge, 1992.

[29] Zhao, Jia; Zhang, Xianke, Coefficients of ternary cyclotomic polynomials. J. Number Theory 130 (2010), no. 10, 2223-2237. 